\title{
Achieving the Maximal Throughput Via a Collision-aware DCF
}

\author{
Hongyuan Chen \\ School of Electronic and Optical Engineering \\ Nanjing University of Science and Technology (NJUST) \\ Nanjing 210094, People's Republic of China \\ Email: chy@mail.njust.edu.cn
}

\begin{abstract}
DCF adopts a binary backoff procedure in order to avoid the further collision. In a real wireless channel, both a channel bit error and a collision can cause a data frame loss. However, DCF always assumes that a data frame loss is due to the collision. In fact, a channel bit error should not cause the backoff window double. In this paper, we propose a Collision Aware (CA) DCF to differentiate between channel error and collision. The CA-DCF introduces a $\mathrm{CA}$ frame which is so small size that it is loss only due to collision. Through theoretical analysis, an optimal backoff window is deduced and the CA-DCF adopts the fixed optimal window as the backoff window. NS-2 simulation results show that the CA-DCF is an effective scheme.
\end{abstract}

Keywords-IEEE 802.11, CSMA/CA, DCF, Collision Aware

\section{INTRODUCTION}

The Distributed Contention Function (DCF) is the main medium access scheme in the MAC layer of IEEE 802.11. Specifically, the DCF employs a Carrier Sense Multiple Access with Collision Avoidance (CSMA/CA) with a binary exponential backoff procedure. The Hot-Pots established by the IEEE 802.11 Access Points have covered almost hot zones, such as the university, the coffee shop, the departments etc. The business success on the WLAN promotes the industry and academics to give so much energy on the target how to improve the performance of WLAN. The higher data rate in physical layer [1] and more robust quality of services [2] are the ways to reach the target. Out of question, the throughput of IEEE 802.11 networks is mainly determined by the DCF scheme. Furthermore, the DCF performance is mainly determined by the backoff procedure.

As to how to improve the performance of DCF, there have so many published articles, but, almost of them were aimed to optimize the contention windows or the exponential backoff. For example, Cali et al. [3] proposed an interesting algorithm to improve the performance of the IEEE 802.11 MAC protocol. Their basic idea is to dynamically assign the optimal contention window size at each station based on the estimation of the number of active stations. Wang et al. [4] introduced a GDCF which halves the contention window size after some consecutive successful transmissions.

In the DCF, after a data frame is transmitted, the fact that a corresponding ACK frame isn't received in a predefined timeout interval shall cause a data frame loss recognition. The DCF assumes that all data frame losses are caused by the data collision when transmitting. As a result, the contention window shall be doubled after a data loss. In fact, in real wireless environment, the data frame loss can be caused by the imperfect wireless channel. If a data frame loss is due to the channel bit error, the DCF should retransmit the data frame as soon as possible but not to double the contention window. Thus, it has to differentiate the two reasons of a data frame loss for the DCF.

Obviously, how to differentiate the collision and bit error is the main key. In this article, we propose a simple scheme to differentiate the two factors. One specific signal frame, collision-aware frame (CA frame) is specifically designed. The special frame size is so small that we can assume only the collision can cause the special frame loss. Thus, the special frame is collision-aware. By counting the loss rate of the CA frame, it can estimate the collision probability. As a result, the number of the competing stations can be estimated. Then, we can use one fixed backoff window to replace the binary exponential backoff. The fixed backoff window is the optimal window size which is deduced from the estimated station number. We call the scheme as collision-aware DCF (CA-DCF). In a short word, the CA-DCF adds a new CA frame which is used to estimate the station number and deduce the optimal backoff window. Then, the CA-DCF adopts the optimal backoff window as the backoff window. In the next sections, we introduce the CA-DCF and give the theoretical and simulation results to validate it.

\section{CA-DCF OVER $802.11 \mathrm{~N}$}

As illustrated in Figure.1, the CA-DCF introduces two new specific frame: $\mathrm{CA}$ frame and CA-ACK frame. Before transmitting one MPDU, the station uses the handshake of CA and CA-ACK to obtain the opportunity of the channel access. The handshake of CA and CA-ACK adopts the CSMA/CA. If the CA can be successfully transmitted, the contention window shall be reset to the initial window size for the next new contention resolution. If the CA-ACK doesn't received, it shows that the $\mathrm{CA}$ is fail to be transmitted. Then, the contention window will be double for the $\mathrm{CA}$ retransmission.

For each MPDU, regardless of its transmission status (successful or fail), the CA and CA-ACK has to be transmitted firstly. Thus, each time of MPDU transmission is a new contention resolution procedure, that is, the backoff procedure starts with the initial contention window. As to 
the data sturcture of the CA and CA-ACK, it contains the PHY header and MAC header plus CRC bytes without any payload bits. The management bits of MAC header can indicate which frame is either CA or CA-ACK.

The transmission data rate of the CA (CA-ACK) may be random only if the CA (CA-ACK) can be transmitted in one symbol. It is known that the 802.11n PHY layer uses the OFDM modulation. The frame size of the CA (CA-ACK) is so small that it can transmitted within one OFDM symbol. At the same time, The CA (CA-ACK) doesn't need one full OFDM symbol due to its small frame size and high PHY layer data rate. For example, if the data rate is $600 \mathrm{Mbps}$, the real transmission time of CA is only ten percentage of one OFDM symbol. But, in this one symbol, it doesn't transmit other data frames. That is, the CA frame must fully occupy one entire symbol. In order to fully utilize one symbol, it can duplicate the payload of the CA (CA-ACK) serval times when transmitting. This can make the transmission of CA (CA-ACK) more robust. The CA loss due to the channel bit error shall be greatly degraded and even near to zero. Further, it makes our assumption more reasonable.

\section{ANALYTICAL MODEL}

First, we assume the CA-DCF adopts the binary exponential backoff window. That is, for the first transmission, the initial window size is adopted. After a failed of transmission of CA frame, the backoff window is double. If the CA frame is successfully transmitted, the backoff window is reset to the initial window for the next round of contention. Based on the above CA-DCF with binary backoff window, we develop a Markov model to deduce the optimal window size.

Since the CA-DCF also adopts the CSMA/CA, it has the same Markov chain as proposed in [5] by taking into account the channel bit error. A bi-dimensional Markov chain $(\mathrm{s}(\mathrm{t}) ; \mathrm{b}(\mathrm{t}))$ is developed for the tagged station (STA). $b(t)$ and $s(t)$ represent the value of backoff counter and the backoff stage at model slot time $t$, respectively. The Markov chain is shown in Figure.2.

The network consists of $n$ contending STAs and each STA always has data frame awaiting for transmission. Since the channel bit error has to be considered for the MPDU transmission, in this article a Gaussian channel is assumed where each bit of one MPDU has the same bit error probability (BER) and bit errors are identically and independently distributed over the whole MPDU.

Let $\mathrm{W}=\mathrm{CW}_{\min }+1, \quad \mathrm{~W}_{\max }=\mathrm{CW}_{\max }+1$ and $\mathrm{m}=\log _{2}\left(\mathrm{~W}_{\max } / \mathrm{W}\right) . \quad \mathrm{CW}_{\min }$ and $\mathrm{CW}_{\max }$ denote the initial contention window and the maximum contention window, respectively. Let $\mathrm{CW}_{\mathrm{i}}$ be the contention window in the $\mathrm{i}$-th retransmission attempt of the $\mathrm{CA}$ frame and $\mathrm{W}_{\mathrm{i}}=\mathrm{CW}_{\mathrm{i}}+1$. Based on the backoff procedure, it obtains $\mathrm{W}_{\mathrm{i}}=2^{\mathrm{i}} \mathrm{W}$; for $0 \leq \mathrm{i} \leq \mathrm{m}-1$ and $\mathrm{W}_{\mathrm{i}}=2^{\mathrm{m}} \mathrm{W}$; for $\mathrm{m} \leq \mathrm{i} \leq \infty$. In the Markov chain, the only non-null one-step transition probabilities are:

$$
\begin{cases}P\{j, k \mid j, k+1\}=1, & k \in\left[0, W_{j}-2\right], j \in[0, m] \\ P\{j, k \mid j-1,0\}=p_{f} / W_{j}, & k \in\left[0, W_{j}-1\right], j \in[1, m] \\ P\{0, k \mid j, 0\}=\left(1-p_{f}\right) / W, & k \in\left[0, W_{-1}-1\right], j \in[0, m] \\ P\{m, k \mid m, 0\}=p_{f} / W_{\max }, & k \in\left[0, W_{\max }-1\right]\end{cases}
$$

The $\mathrm{p}_{\mathrm{f}}$ in Figure. 2 represents the probability that the CA frame is failed to be transmitted. Since the CA loss is mainly due to the collision, we can assume that the CA frame is free to the channel bit error. Then, the $\mathrm{p}_{\mathrm{f}}$ shall be the collision probability. Once there are more than one STA transmitting the CA frame at the same time, the collision occurs. Let $\tau$ be the probability that a STA transmits in a randomly chosen slot time, then we have $\mathrm{p}_{\mathrm{f}}=1-(1-\tau)^{\mathrm{n}-1}$.

Based on the chain regularities, we can solve the Markov chain and obtain the $\tau$ by imposing the normalization condition.

$$
\tau=\frac{2}{1+W+p_{f} W \sum_{j=0}^{m-1}\left(2 p_{f}\right)^{j}}
$$

For the transmission of CA frame in a give slot time, there are three status. Firstly, the STA is during the backoff counter decrement. So, there are no transmission in this one slot. Secondly, a collision of CA frames happens in this one slot. Thirdly, the STA successfully transmits the CA frame in this one slot. In order to compute the throughput, it has to analyze the duration of the above each status and corresponding probability.

Before going into the next step, we have to give some denotations. Let $\delta, \mathrm{T}_{\mathrm{MPDU}}$ and $\mathrm{T}_{\mathrm{ACK}}$ denote duration of an empty slot time, the time to transmit one MPDU frame and the time to transmit an ACK frame, respectively. Correspondingly, $\mathrm{T}_{\mathrm{CA}}$ and $\mathrm{T}_{\mathrm{CAACK}}$ are the times to transmit one CA frame and one CA-ACK frame, respectively. After transmitting the $\mathrm{CA}$ frame, the $\mathrm{CA}$ frame can be assumed to loss if the CA-ACK isn't received within an CA-ACK timeout. The CA-ACK timeout is equal to the sum of a SIFS interval and CA-ACK transmission time plus an additional DIFS (i.e. SIFS $+\mathrm{T}_{\mathrm{CAACK}}+\mathrm{DIFS}$ ).

For the first status, its duration shall be the empty slot time $\mathrm{d}$ since all STAs are waiting for transmission. For the second status, a collision of CA frame only cause the loss of $\mathrm{CA}$ frame and the corresponding CA-ACK timeout. For the third status, there are two cases needed to be considered. Since the CA frame is successfully transmitted, the MPDU must be sent following the handshake of CA and CA-ACK. Due to the imperfect channel, the MPDU can be loss. If the MPDU is successfully transmitted, the duration shall be the time of CA/CA-ACK handshake and one MPDU transmission time plus corresponding ACK time. If the MPDU is fail to be transmitted, the duration shall be the time of CA/CA-ACK handshake and one MPDU transmission time plus corresponding ACK timeout $\left(\mathrm{ACK}_{\text {timeout }}\right)$. Let $\mathrm{T}_{\mathrm{c}}$ be the duration of one CA collision, $\mathrm{T}_{\mathrm{s}}$ be the duration of one successful MPDU transmission and $\mathrm{T}_{\mathrm{f}}$ be the duration of one failed MPDU transmission. Then 


$$
\left\{\begin{array}{l}
T_{c}=T_{C A}+S I F S+T_{C A A C K}+D I F S \\
T_{s}=T_{C A}+T_{C A A C K}+T_{M P D U}+T_{A C K}+3 S I F S+D I F S \\
T_{f}=T_{C A}+T_{C A A C K}+T_{M P D U}+2 S I F S+A C K_{\text {timeout }}+D I F S
\end{array}\right.
$$

Let $P_{c}$, Ps and $P_{f}$ be the corresponding probability of $T_{c}$, Ts and $T_{f}$, respectively. Let $p_{e}$ be the probability that a MPDU (with size $L_{\text {data }}$ ) and/or ACK frame (with frame size $\left.\mathrm{L}_{\text {ack }}\right)$ suffer errors. $\mathrm{p}_{\mathrm{e}}=1-(1-\mathrm{BER})^{\mathrm{Ldata}}(1-\mathrm{BER})^{\text {Lack }}$.

$$
\left\{\begin{array}{l}
P_{c}=1-(1-\tau)^{n}-n \tau(1-\tau)^{n-1} \\
P_{s}=n \tau(1-\tau)^{n-1}\left(1-p_{e}\right) \\
P_{f}=n \tau(1-\tau)^{n-1} p_{e} \\
P_{i}=(1-\tau)^{n}
\end{array}\right.
$$

Let $\mathrm{P}$ denote the effective payload size of one MPDU. Thus, the throughput can be expressed as

$$
S=\frac{P_{s} \cdot P}{P_{i} \cdot \delta+P_{c} T_{c}+P_{s} T_{s}+P_{f} T_{f}}
$$

\section{Optimal CONTENTION Window}

The CA frame can be used as a pilot to estimate the number of contending stations. Since the loss of the CA frame is caused only by the collision, it is able to estimate the collision probability. Given an estimated interval, the collision probability is estimated by

\section{$\widehat{p_{f}}=\frac{\text { the number of the failed } C A}{\text { the number of } C A \text { in the estimated interval }}$}

Based on the equation (5) and the similar analysis proposed in [6], we can also obtain the optimal $\tau_{\text {opt }}=1 /\left(n \cdot \sqrt{T_{c} / 2 \delta}\right)$ by taking $\mathrm{T}_{\mathrm{s}}=\mathrm{T}_{\mathrm{f}}$ due to $\mathrm{ACK}_{\text {timeout }}$ $=\mathrm{T}_{\mathrm{ACK}}+\mathrm{SIFS}$. Through the estimated $\overline{p_{f}}$, the number $\bar{n}$ of contending stations can be estimated by

$$
\widehat{p_{f}}=1-\left(1-\frac{1}{\widehat{n} \cdot \sqrt{T_{c} / 2 \delta}}\right)^{\widehat{n}-1}
$$

The CA-DCF uses the optimal contention window $W_{\text {opt }}$ but not binary exponential window. For a fixed backoff window size, we have $\tau=2 /(\mathrm{W}+1)[6]$. Finally, it obtains $W_{\text {opt }}=2 / \tau_{\text {opt }}-1$.

\section{PERFORMANCE EVALUATION}

We implement the CA-DCF in the simulation tool NS-2. The simulation results are illustrated in the Figure.3. Except for the special statements, all the PHY and MAC parameters are compliant with the 802.11n standard [1]. Three [7] scenarios, ideal channel $(\mathrm{BER}=0)$, moderate channel error $\left(\mathrm{BER}=10^{-6}\right)$ and very noisy channel $\left(\mathrm{BER}=10^{-5}\right)$, are investigated. Taking into account the frame aggregation functionality of $802.11 \mathrm{n}$, the payload size of each MPDU is 8000 Bytes. The CA-DCF can significantly improve the throughput in almost of network scenarios. This is reasonable due to the fact that the optimal window can achieve the best balance between the backoff waiting time and collision probability. We have to note that the enhanced gap for the low channel error $\left(\mathrm{BER}=10^{-6}\right)$ is greater than that for the high channel error $\left(\mathrm{BER}=10^{-5}\right)$.

\section{CONCLUSIONS}

This paper introduces a collision aware DCF CA-DCF. The CA-DCF adds a CA frame to differentiate between the collision and channel bit error. Since the CA frame is loss only due to the collision, it can estimate the contending station number by counting the CA frame loss probability. As a result, an optimal backoff window is deduced and the CA-DCF adopts the fixed optimal window size as the backoff window. We implement the CA-DCF in NS2 simulation tool and run the CA-DCF over 802.11n. Simulation results show that the CA-DCF is an effective scheme to enhance the throughput.

\section{ACKNOWLEDGE}

This work was supported by ZiJing Scholar Plan of NJUST (No.AB41375).

\section{REFERENCES}

[1] IEEE, Part 11: Wireless lan medium access control (MAC) and physical layer (PHY) specifications: Amendment 5: Enhancements for higher throughput. IEEE 802.11n, Sept, 2009

[2] IEEE, Part 11: Wireless medium access control (MAC) and physical layer(PHY) specifications: Amendment 8: Medium access control (MAC) quality of service enhancements. IEEE 802.11e, Sep, 2005.

[3] Cali, F., Conti, M., and Gregori, E., Dynamic tuning of the IEEE 802.11 protocol to achieve a theoretical throughput limit. IEEE/ACM Trans. Netw., 8(6), Dec, pp. 785-799, 2000.

[4] Wang, C., Li, B., and Li, L., A new collision resolution mechanism to enhance the performance of IEEE 802.11 DCF. IEEE Trans. Veh. Technol., 53(4), Apr., pp. 1235-1246, 2004.

[5] Ni, Q., Li, T., Turletti, T., and Xiao, Y., Saturation throughput analysis of error-prone 802.11 wireless networks. Wiley J. Wireless Commun. and Mobile Comput., 5(8), Dec., pp. 945-956, 2005.

[6] Bianchi, G., Performance analysis of the IEEE 802.11 distributed coordination function. IEEE J. Sel. Areas Commun., 18(3), Mar., pp. 535-547, 2000. 


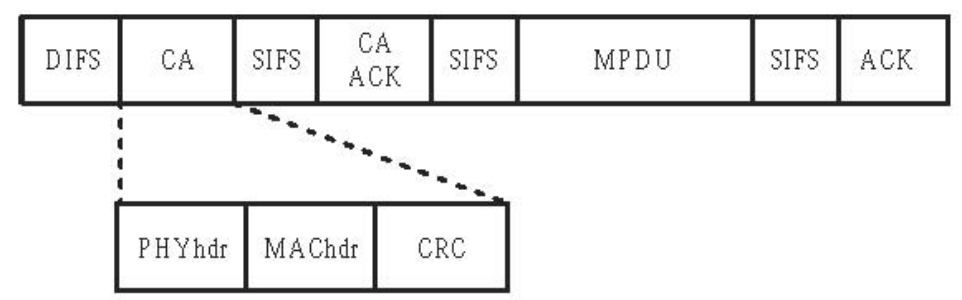

Figure 1. A Typical data sequence when CA-DCF is adopted

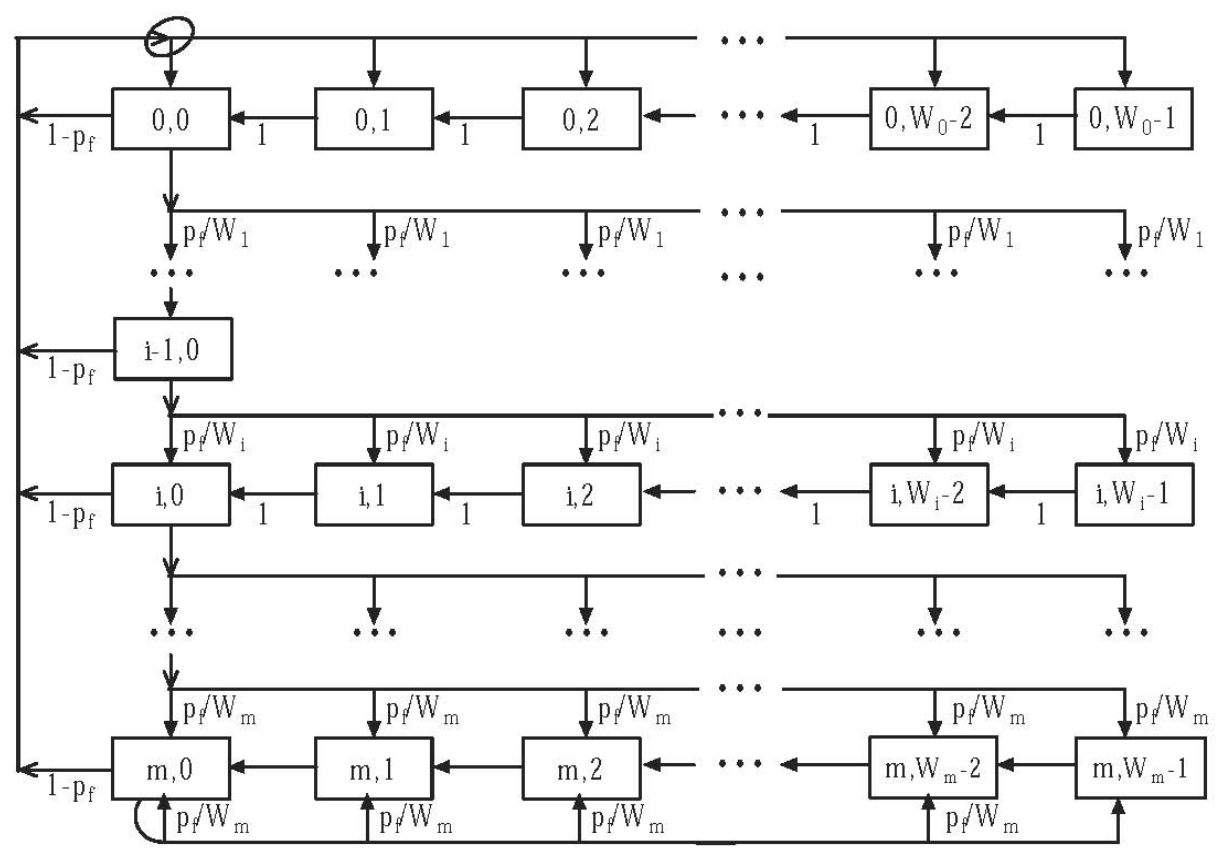

Figure 2. State transition diagram for CA-DCF

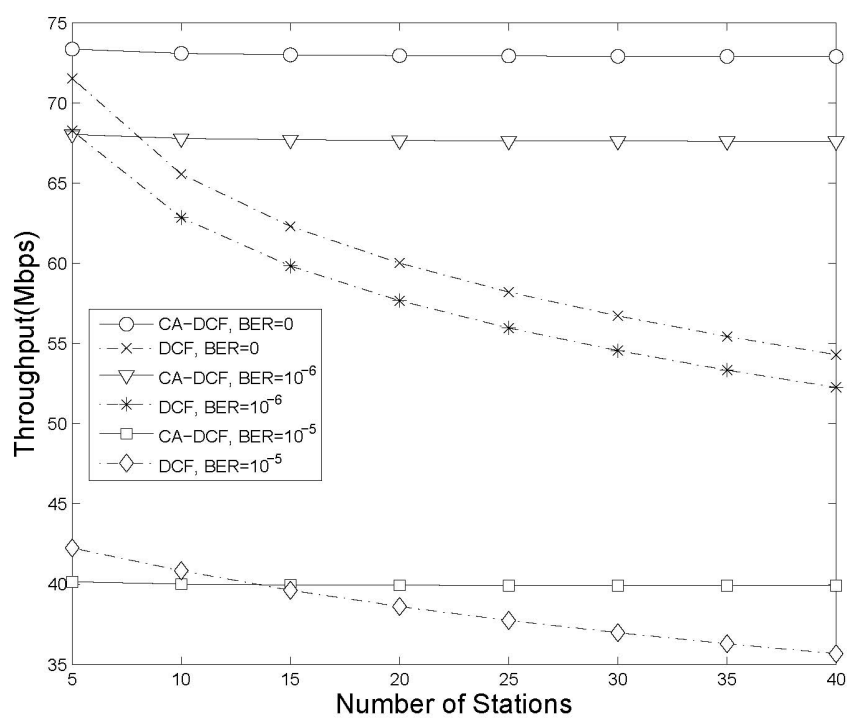

Figure 3. Model Validation: Rate $=108 \mathrm{Mbps}, \mathrm{CW}_{\min }=7, \mathrm{CW}_{\max }=1023$ 